\title{
A Projection Pursuit Based Risk Assessment Method in Mobile Ad hoc Networks
}

\author{
${ }^{1}$ Fu Cai, ${ }^{1}$ Liu Ming*, ${ }^{2}$ Chen Jing, ${ }^{3}$ Zhang Li, ${ }^{4}$ Xiao-Yang Liu \\ ${ }^{1}$ College of Computer Science \& Technology, Huazhong University of Science \&Technology, Wuhan, 430074,Chinas \\ ${ }^{2}$ School of Computer, Wuhan University, WuHan City, China \\ ${ }^{3}$ Department of Computer Science, School of Computing, National University of Singapore, Singapore \\ ${ }^{4}$ Department of Computer Science and Engineering, Shanghai Jiao Tong University, China \\ E-mail: liuming2008@gmail.com,stand_fucai@126.com
}

\begin{abstract}
Establishing high performance cooperation and estimating nodes' risk level in mobile ad hoc networks (MANETs) are currently fundamental and challenging due to the inher ent characteristics of MANETs, such as the highly dynamic topology and the absence of an effective security mechanism. Trust based assessment methods were recently put forward but presumed restrictions to the data samples or presumed weights for node's attributes are required. In this paper, Projection Pursuit bas ed Risk Assessment (PPRA), is proposed to analyze node's creditability. As projection pursuit turns high-dimensional node properties to low-dimension space, all nodes' risk levels could be clustered effectively and accurately. Projection index, the same as judgment index of clustering consequence, is utilized to reveal the b ehavior of different nodes. By maximizing projection ind ex through Genetic Algor ithm (GA), optimal projection direction is obtained, and then the projection values of e ach node could be calculated. Finally, the results in one-dimension or two- dimension projection space show that our me thod is more efficient and practical than traditional methods.
\end{abstract}

Keywords: project pursuit, genetic algorithm, risk assessment, projection direction, projection index.

\section{Introduction}

Mobile ad hoc n etworks (MANETs) a re co mplex d istributed systems that can dynamically self-organize into "ad-hoc" net work topologies with arb itrariness and temporality, which allows people and devices seamlessly net worked together i $\mathrm{n}$ ar eas wi th $\mathrm{n}$ o pre-deployed infrastructure Ref.1. Si nce nodes $m$ ay $n$ ot reside i $n$ physically protected places, they $m$ ay fall unde $r$ attackers' control. Due $t$ o the broadcasting nature of wireless channels, message eavesdropping and injection are possible. Thus, the security issue of MANET s is a difficult problem. Howev er, classic secu rity so lutions b ased on certification auth orities and on-line serv ers are in applicable because of the absence of infrastructure. Therefore the design of a new and effective security $m$ echanism for MANETs is quite important and necessary.

Currently a variety of as sessment models are put forward, most of which reflect the risk level of communication through trust or reputation evaluation. Generally, these m ethods could be clas sified into $t$ wo categories: probability-based and fuzzy theory-based.

Many works relate closely with $\mathrm{p}$ robability-based models. B eth and his resea rch $g$ roup $p$ ut forward an experience-and-probability-based trust $\mathrm{m}$ odel in Ref.2 . Authors in Ref.3 ad opted the beta distribution probability method to obtain the trust relationship according to a basic principle, $n$ amely the po sterior probability distribution of the node action is subject to beta distribution. In Ref.4, trust was derived from evidence theory. Ref.5

${ }^{*}$ Corresponding author 
and 6 applied fuzzy theory to conceptualize the subjectivity and uncertainty of tru st. Current assessment models have made impressive progress. However, the complex ch aracteristics of M ANETs still d eserve a lo $t$ of concern.

(i) The multiple-attribute and multiple-polarity of the sam ple d ata: wh en con sidering the trust lev el of a node, the communication characters (e.g., packet losing rate) and physic attributes (e.g., signal intensity) should also $b$ e in cluded. Some attri butes are better wh en the data si ze g rows 1 arger, while ot hers are opposite. We call this multiple-polarity. Attributes change temporally which leads to a high dimension space. Current assessment models lack effective mechanisms to directly unveil th e informatio $\mathrm{n}$ with in th is high-dimension $\mathrm{d}$ ata, thus assessing nodes' trust level is challenging.

(ii) Temporal ch aracteristic an $\mathrm{d}$ the reliab ility of trust evaluation: the assessm ent is a dynam ic and complex process. We nee $d$ to process comprehensively regarding each stage, without ignoring the temporal characteristic. The stag es of trust estab lishment, feed back and adjustment should be able to process direct and indirect information. Current evaluation systems deal with the data using prob ability or exp erience, which suffer the problem of bad expansibility. Moreover, they cannot 'sense' slight abnormal attributes which leads to unreliability.

(iii) Uncertainty of ev aluation subj ect: th is in cludes the needs, the e xperience and the knowledge of the s ubject. E .g., c urrent e valuation sy stem uses $t$ he same s trategy to $d$ eal with $d$ ifferent requ irements. In general, if the subject lacks experience and knowledge, evaluation methods will lead to un certain even con tradictory res ults. Therefore, we nee $d$ more objective methods to reduce the negative impact brought by subjective factors.

(iv) Unpredictability an d d iversity of attack s: due to its self-organization, dynamic routing and open wireless channels, MANETs are vulnerable to various kinds of attacks, e.g., the black hole attack and rel atively covert wormhole attack. Some traditional evaluation mechanisms are based on particular distribution principle and others use fun ctions wh ich lack eno ugh mathematic basis or are limited to some particular attacks. We need to seek for new evaluation methods to provide all-aspect basis.

Besides, $t$ he methods a bove al 1 have $s$ ome mentioned dr awbacks. Fir st, nodes $\mathrm{w}$ ith the dynamic an $\mathrm{d}$ transitional p roperties, such as malicious no des which are al ways sel f-protective, deviate the behaviors from the statistical 1 aw. Using the probability theory will reduce the accuracy of assessment. Second, a huge sample data is required when presumed distribution is app lied; otherwise the result would be unconvincing. Third, the fuzzy theory requires the behavior of the node to satisfy their presumed patterns.

To solve the above problems, PPRA is prop osed. It first maps multi-dimensional data in to low-dimensional manifolds for visual inspection, and then clusters nodes according to their projection val ues. Proje ction i ndex which is sub jected to the projection direction, as th e judgment of clustering e ffect, sh ould be m aximized. Projection pursuit (PP) algo rithm ${ }^{[7-10]}$ searches all pos sible projection directions in order to find the optimal projection direction $t$ hat $m$ aximizes $t$ he projection index.

Due to in complete inform ation and no presu med pattern of nodes' be havior in the M ANETs, m any aspects must be considered to reveal the risk lev el. So a high-dimension matrix, called ind icator m atrix, is con structed. Through analyzing the sample data of the indicator matrix, Genetic Algorithm (GA) is employed to search for the optimal projection direction by maximizing the projection index. PPRA model is a "data-driven" model an d risk lev el is ach ieved thr ough an alyzing sample data till the conve rgence. No presumed requirements are needed. Through experiment, PPRA model is proved to be robust, which can endure noisy of sample data to some degree.

The structure of the paper is as follows: Section 2 provides some basic knowledge about Projection Pursuit Theory. Section 3 introduces the detailed procedures of the assessment method based on PP. Section 4 demonstrates experimental analysis and comparisons with other m echanisms. Pe rformance analysis is i $\mathrm{n}$ Section 5 and Section 6 concludes this paper.

\section{Projection Pursuit \& Genetic Algorithm}

Projection $\mathrm{Pu}$ rsuit (PP) is mainly $\mathrm{u}$ sed for an alyzing high-dimension data, especially non-normal population. The main idea of PP is: $p$ roject high-dimension data to low-dimension through the optimum proje ction direction which could best reflect the data structure and characteristic. While traditional methods treat time coordinate as probability or $\mathrm{p}$ articular $\mathrm{d}$ istribution function, 
projection pu rsuit uni fies di fferent attribute values at different time.

A sim ple $g$ enetic alg orithm (SGA) is a numerical search technique used to find $t$ he exact or approximate solution for optimization problems. In SGA, a population is the abstract representation of candidate solutions. Besides, it evolves toward better solutions each step and models 1 oosely o $\mathrm{n}$ the principles of nat ural sel ection: employing a population of individuals that undergo selection in the presence of va riation-inducing operators such as mutation and crossover.

In the following, we provide som e basic conce pts and definitions.

\section{(I) Overall Data Dispersion}

The h igh-dimension attrib ute $m$ atrix $Y$ is indicated as $Y=\left(y_{1}, y_{2}, \ldots, y_{n}\right)$. Ov erall data dispersion $S(a)$ ind icates the rate of deviation from the average:

$$
S_{y}=\sqrt{\frac{\sum_{i=1}^{n}\left((Y(i)-E(Y))^{2}\right.}{n-1} .}
$$

\section{(II) Local Data Density}

$D(a)$ i ndicates t he distribution of t rust e valuation values in one-dimension space.

$$
D_{y}=\sum_{i=1}^{n} \sum_{j=1}^{n}\left(R-r_{i j}\right) U\left(R-r_{i j}\right) .
$$

Where, $r_{i j}$ indicates the dista nce between evaluation values. Fo $\mathrm{r}$ o ne-dimension, $r_{i j}=\left|y_{i}-y_{j}\right|$, a nd $\mathrm{f}$ or tw odimension, $r_{i j}=\sqrt{\left|Y_{i}-Y_{j}\right|^{2}+\left|Y_{i}^{\prime}-Y^{\prime}{ }_{j}\right|^{2}} . R$ is the density window width, a nd $\mathrm{i}$ ts sel ection $\mathrm{s}$ hould sec ure $\mathrm{t}$ hat $\mathrm{t}$ he amount of nodes in the window a re not too little and they will not grow too high with the increase of $n$.

Theorem 1: The projection density window width $R$ satisfies the following equation: $r_{\max }<R \leqslant p$. There are $n$ projection samples, the dimension is $p$ and $r_{\text {max }}$ indicates the maximum range between projections.

Demonstration: Based on the definition of local data density

$$
\begin{aligned}
D_{y}= & \sum_{i=1}^{n} \sum_{j=1}^{n}\left(R-r_{i, j}\right) U\left(R-r_{i, j}\right) \\
= & \left.\sum_{i=1}^{n}\left(R-r_{i, 1}\right) U\left(R-r_{i, 1}\right)+\left(R-r_{i, 2}\right) U\left(R-r_{i, 2}\right)+\ldots+\left(R-r_{i, n}\right) U\left(R-r_{i, n}\right)\right] \\
= & \left(R-r_{1,1}\right) U\left(R-r_{1,1}\right)+\left(R-r_{1,2}\right) U\left(R-r_{1,2}\right)+\ldots+\left(R-r_{1, n}\right) U\left(R-r_{1, n}\right) \\
& +\left(R-r_{2,1}\right) U\left(R-r_{2,2}\right)+\left(R-r_{2,2}\right) U\left(R-r_{2,2}\right)+\ldots+\left(R-r_{2, n}\right) U\left(R-r_{2, n}\right)+ \\
& \ldots+\left(R-r_{n, 1}\right) U\left(R-r_{n, 2}\right)+\left(R-r_{n, 2}\right) U\left(R-r_{n, 2}\right)+\ldots+\left(R-r_{n, n}\right) U\left(R-r_{n, n}\right)
\end{aligned}
$$

Since $\mathrm{R}>0$, and $i=j$, we have

$$
r_{i j}=r_{j i}=0,\left(R-r_{i j}\right) * U\left(R-r_{i j}\right)=R,
$$

then we can get

$$
\begin{aligned}
D_{y}= & n R+2 \sum_{i=2}^{n}\left(R-r_{1 i}\right) S\left(R-r_{1 i}\right)+2 \sum_{i=2}^{n}\left(R-r_{2 i}\right) S\left(R-r_{2 i}\right) \\
& +2 \sum_{i=n-1}^{n}\left(R-r_{n-2, i}\right) S\left(R-r_{n-2, i}\right)+2\left(R-r_{n-1, n}\right) S\left(R-r_{n-1, n}\right),
\end{aligned}
$$

assume $r_{\max }=\max \left(r_{j i}\right)$, only whe $\mathrm{n} R>r_{\max }$, satisfying $S\left(R-r_{i j}\right)=1, D_{y}$ can be $t$ he $\mathrm{m}$ aximum, and the greater

$\mathrm{R}$, the greater $D_{y}$.

On t he other han $\mathrm{d}$, $\|X\| \leq 1,\|a\| \leq 1$, based on the actual physical meaning of the clustering projection $t$ o one-dimension s pace, $0 \leq a \leq 1 Y=\alpha^{T} X$, so $0 \leq Y_{i} \leq p$, and $r_{i j}=\left|Y_{i}-Y_{j}\right|$, so $r_{i j} \leq p$.

So $r_{\max }<R \leq p$ comes into existence.

\section{(III) Projection Index}

Projection index is used to ju dge whether a projection direction is a $\mathrm{m}$ eaningful $\mathrm{t}$ arget $\mathrm{f}$ unction. I $\mathrm{n}$ our scheme, we use the classic projection index to optimize projection direction. T hat i s: $Q(a)=S(a) * D(a)$, w here $S(a)$ i ndicates ove rall dat a di spersion, $D(a)$ indicates partial data density, $a$ indicates the projection direction.

\section{(IV) Optimum Projection Direction}

Given the high-dimension node attribute matrix, the projection i ndex fun ction will ch ange with pro jection direction. We ho pe to find out the opt imal projection direction wh ich can rev eal u ltimately the attributes' characteristic. The optimal direction can ul timately reflect the hi gh-dimension data st ructure, a nd then we analyze the projecte $d$ one-dimension data. W e defi ne projection direction searching as following:

$$
\text { Opt } a:\left\{\begin{array}{c}
\max : Q(\alpha)=\mathrm{S}(\alpha) \mathrm{D}(\alpha) \\
\text { s.t. } \quad \sum_{i=1}^{p} a_{i}=1
\end{array}\right. \text {. }
$$

Thus, trust evaluation convert to this problem: solving complex nonlinear op timum by optimizing parameters. An appropriate method to search optimal projection direction is the real code d genetic al gorithm (R AGA) ${ }^{[11-12]}$. Given the obtained optimal direction and the projection index, an alysis of u nknown nodes d epends on simple matrix calculation.

\section{(V) Visualized Clustering Projection}

In general projection pursuit, we usually project data to one-dimension space to quantize pr ojection res ults. While in actual analysis, we need to project data to twodimensional or three-dimensional space. System administrator can directly judge whether a node is abnormal which streng ths th e $d$ iscernment. We call th is two - 
dimensional and three-dimensional projection as visualized clustering projection. Vi sualized clustering projection owns two impressive characters: information loss is fewer and the result is visualized.

Choose two one-dimension projection vectors, $a, a$, $\in R^{p}$, calculat e projection values res pectively, $Y=a^{T} X$, $Y^{\prime}=a^{, T} X$. These two vectors can maximize the am ount of i nformation of joint distribution of $Y$ a nd $Y^{\prime}$. The purpose of projection is to find the nonlinear structure of original data, so $Y$ and $Y^{\prime}$ should be irrelevant which requires $a$ and $a^{\prime}$ to b e or thogonal, su ch that $a^{T} a^{\prime}=0$, $a^{T} a=1, a^{, T} a^{\prime}=1$. The distance of sample projections is:

$$
r_{i j}^{\prime}=\sqrt{\left|Y_{i}-Y_{j}\right|^{2}+\left|Y_{i}^{\prime}-Y_{j}^{\prime}\right|^{2}} \text {. }
$$

In two-dimension projection, the projection de nsity window width $R$ is a variable to be determined, from theorem 1 we have the following inference.

Corollary 1: There ar e $n$ p rojection sam ples, the dimension is $p$ and $r_{\max }$ indicates the $\mathrm{m}$ aximum range between projections. The p rojection density wi ndow width $R$ in two-dimension space satisfies the following equation: $r_{\max }<R \leq 2 \sqrt{2} p$.

Demonstration: From theorem 1 we can get that

$$
\begin{aligned}
D_{y}= & n R+2 \sum_{i=2}^{n}\left(R-r_{1 i}\right) S\left(R-r_{1 i}\right)+2 \sum_{i=2}^{n}\left(R-r_{2 i}\right) S\left(R-r_{2 i}\right) \\
& +2 \sum_{i=n-1}^{n}\left(R-r_{n-2, i}\right) S\left(R-r_{n-2, i}\right)+2\left(R-r_{n-1, n}\right) S\left(R-r_{n-1, n}\right)
\end{aligned},
$$

assume $r_{\max }=\max \left(r_{j i}\right)$, only whe $\mathrm{n} P r_{\max }$ can satisfy $S\left(R-r_{i j}\right)=1, D_{y}$ can be the $\mathrm{m}$ aximum, and $\mathrm{t}$ he greater R, the greater $D_{y}$.

On the other hand, $\|X\| \leq 1,\|a\| \leq 1,\left\|a^{\prime}\right\| \leq 1$, Based on the actual physical meaning of the clustering projection, that $-1 \leq a \leq 1,0 \leq a^{\prime} \leq 1$ and from $t$ he pr ojection $Y=\alpha^{T} X$, then $-p \leq Y_{i} \leq p,-p \leq Y_{i}^{\prime} \leq p$, that is :

$-p \leq Y_{i}-Y^{\prime}{ }_{i} \leq p \quad$, and $\sin$ ce $r_{i j}=\sqrt{\left|Y_{i}-Y_{j}\right|^{2}+\left|Y_{i}^{\prime}{ }_{i}-Y^{\prime}{ }_{j}\right|^{2}}$, then, $r_{i j} \leq 2 \sqrt{2} p$.

So $r_{\max }<R \leq 2 \sqrt{2} p$ comes into existence.

\section{Projection Pursuit Model}

The purpose of PPRA Model is to assess e very node's risk level. PPRA can add time character which is appreciated because some attack behaviors seem well in most of the time and only through temporal analysis they can be detected. Secondly, the indicator matrix is constructed. Finally, risk level clustering is assessed by calculating every no de's projection $\mathrm{v}$ alue. In this $\mathrm{p}$ aper, o ne- dimension projection and two-dimension projection are presented.

\subsection{Elementary principle of PP model}

\subsubsection{Constructing indicator matrix}

Let $S_{i j}^{t}$ be the property value for indicator $Y_{j}$ in node $X_{i}$ at the time $t(i=1,2, \ldots, n, j=1,2, \ldots, m, t=1,2, \ldots, T)$.

Then the indicator matrix, in symbol $S$, is as follows:

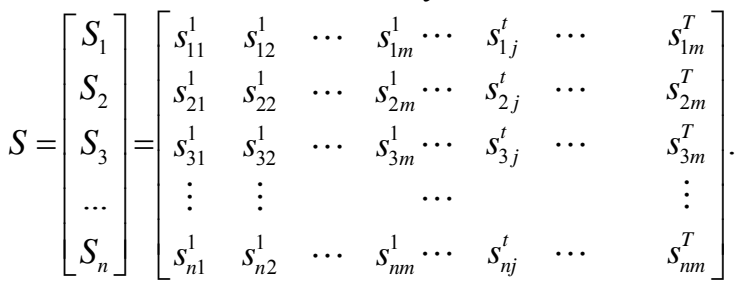

\subsubsection{Normalizing}

To eliminate this negative impact of indicators' unit, normalization of $S_{i j}^{t}$ is necessary. If indicator $j(j=1,2 \ldots$ $m$ ) is positive-effect, then,

$$
s_{k j}^{i}=\frac{s_{k j}^{i}-\min _{k}\left(s_{k j}^{i}\right)}{\max _{k}\left(s_{k j}^{i}\right)-\min _{k}\left(s_{k j}^{i}\right)} .
$$

Otherwise,

$$
s_{k j}^{i}=\frac{\max _{k}\left(s_{k j}^{i}\right)-s_{k j}^{i}}{\max _{k}\left(s_{k j}^{i}\right)-\min _{k}\left(s_{k j}^{i}\right)} .
$$

After normalization, indicator characteristic matrix $S$ ranges from 0 to 1 . Normalization can reduce the influence of different unit of each at tribute, and make ou $\mathrm{r}$ comprehensive analysis possible.

\subsubsection{Constructing projection index}

Projection pursuit is to map high-dimension data onto low dimension through linear co mbination. Let $Z$ be the projection value as follows:

$$
Z_{i}=f(\alpha)=\sum_{j=1}^{m}\left(\mathrm{a}_{j} * s_{i j}\right) .
$$

Where, $a$ is projection direction. In order to objectively reflect charact er of high dimension, pr ojection pu rsuit regression adopts the sum of a series of ridge function to approximate $r$ egression $\mathrm{f}$ unction. We a dopt $\mathrm{t}$ he su per smooth regression as in Ref.8. The convergence condition, namely, projection index is as follows:

$$
Q(a)=S(a) * D(a)
$$

Where, $S_{z}$ stands for standard deviation of projection $Z, D_{z}$ stands for local density of projection $Z$. As to twodimension projection, projection index is as follows: 


$$
Q(a, b)=S_{z}(a, b) * D_{z}(a, b)
$$

\subsubsection{Optimizing projection index by GA}

Different projection di rections reflect different $d$ ata structure cha racters. Pro jection ind ex s hould be maximized to reach the la rgest differe nce among all nodes.

PPRA model could be described as follows:

$$
\begin{gathered}
\max : Q(\alpha)=S_{z} * D_{z} \\
\text { s.t.: }\left\{\begin{array}{c}
\sum_{i=1}^{40} a_{i}^{2}=1 \\
0 \leq a_{i} \leq 1 \text { for } i=1,2 \ldots 40
\end{array}\right.
\end{gathered}
$$

Where $R$ is a n experiment-driven pa rameter, which is decided by experiment result and $r(i, j)=|z(i)-z(j)|$.

As mentioned above, GA algorithm is employed to solve the problem. Through searching possible solutions in the whole solution space, optimal projection direction $a$ is obtained.

Similar to above, two-dimension projection could be described as follows:

$$
\begin{aligned}
& \text { max: } Q(a, b)=S_{z}(a, b) * D_{z}(a, b) \\
& \text { s.t. : }\left\{\begin{array}{c}
S_{z}(a, b)=S_{z}(a) * S_{z}(b) \\
\sum_{i=1}^{p} a_{i}^{2}=1, \sum_{i=1}^{p} b_{i}^{2}=1 \\
\sum_{i=1}^{p} a_{i} b_{i}=0 \\
0 \leq a_{i} \leq 1 \text { for } i=1,2 \ldots p
\end{array}\right.
\end{aligned}
$$

\subsubsection{Sorting the candidate nodes}

First, let's show that the relationship between node's projection value and its corresponding risk level.

Theorem 2: Through the steps above, the larger the projection value is, the higher the creditability is.

Demonstration: Let us assume one vi rtual $\mathrm{n}$ ode named n ode $V . V$ rep resents the op timal n ode, i.e. at anytime at any attrib ute nod e $\mathrm{V}$ get the best attribu te value. And after the temporary normalization, obviously node $V$ 's attribute value is all 1 .

Because

$$
Z_{i}=f(\alpha)=\sum_{j=1}^{m}\left(\mathrm{a}_{j} * s_{i j}\right) .
$$

And all attribute values of $\mathrm{V}$ node are 1, thus:

$$
Z_{V}=f(\alpha)=\sum_{j=1}^{m}\left(a_{j}\right)=1 .
$$

That means the optimal node's projection value is 1 . So if real node's projection value is greater, the differ- ence betwee $\mathrm{n}$ it and optim al node is sm aller, i.e. this node is better. This proves our conclusion is right.

Therefore, after obtaini ng the projection value o $\mathrm{f}$ each node by the steps above, a final evaluation for these candidate $n$ odes can be achieved. The no de's risk level decreased with the increase of the projection value; meanwhile there still exist more simila rities for specific two nodes when they're more close to eac $h$ other. And the sam e co nclusions c ould be i nferred for tw odimension proj ection. The more si milar to $(1,1)$, the higher the creditability is.

\subsection{Procedures of PPRA model}

The procedures of PPRA are as following:

Step1: selecting key indicators.

Step2: normalizing $S$, and obtaining the $S$ '.

Step3: using $G A$ algorithm to obtain optim al projection direction.

Step4: sorting the candidate nodes.

Once the optimal projectio $\mathrm{n}$ directio $\mathrm{n}$ is obtained, every candidate nod e's proj ection value will be calculated in optimal projection direction. Then after sorting these projection values, we can get reliable and precise results concerning nodes' risk and their similarities.

\section{Risk Assessment Scheme in MANETs}

Theoretical PPRA m odel is showe $\mathrm{d}$ a bove. A real e $\mathrm{x}$ ample in MANET is presented below. However, before carrying out the experiment, de fining key attributes is also of significance. Afte $r$ fulfilling PPRA model, analysis of final result and co mparison between PPRA and other methods are co nducted. We conclude that PPR A model is $\mathrm{m}$ ore s uitable to various network behaviors, not only for its accuracy but also its convenience as well.

\subsection{Defining key attributes}

The selected in dicators should properly $\mathrm{r}$ eflect the behavior characteristics of the nodes in order to make the risk assessment objective and efficient. Establishing basic principle for selection's convenience is necessary.

(I) Accessible. The i ndicator's value $\mathrm{m}$ ust be obtained easily. As data is sampled from real M ANETs, accessibility is basic.

(II) Relative. The chosen indicators should directly reflect node's property. 
(III) Complete. E very node's value should be obtained so that each node could be reflected by the corresponding indicators.

For the risk a ssessment of nodes in M ANETs, as pects incl uding network co mmunication $\mathrm{f}$ eatures (i.e . loss tolerance), physical attr ibutes (i.e. m obility), wireless signal and position should all be taken into consideration. In this pape $\mathrm{r} f$ our indicators are pitched on: transmission speed (TS), losing rate (LR), signal intensity (SI), and signal changing rate (SCR). The categories of these four in dicators a re as follows: P re presents positive-effect, whereas $\mathrm{N}$ re presents negative-effect. And before c onducting e xperiment, basic analysis of selected indicators is prepared.

Table 1. Categories of selected indicators

\begin{tabular}{cll}
\hline TS LR & SI & SCR \\
\hline P N & P N & \\
\hline
\end{tabular}

\subsection{Risk assessment example}

Our experiment platform is NS 2.28. Six nodes are designed, in whic hone attack node and one lowefficiency node are i ncluded. Node 5 loses part of the packet tem porarily and node 6 transm its packet very slow but com pletely. We obtained ten successive m oments' node a ttributes for experiment. For the sa ke of brevity, only losing rate data is s hown in Fig.1. From Fig. 1, the losing rate of node 6 is very high even though it's very stable; and the value of node 5 is small but not stable, more likely to under an attack. Generally speaking, node 1 and node 2 is better than other nodes.

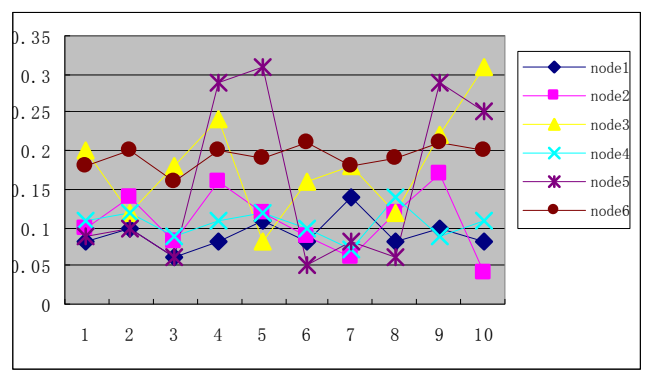

Fig.1. Package losing rate curve

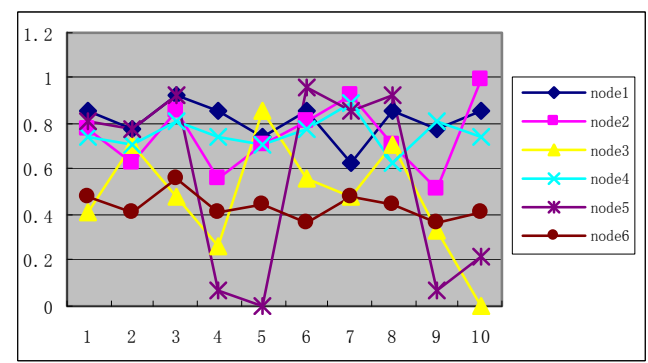

Fig.2. Package losing rate curve after normalization

\section{(I) Construction of indicator matrix}

From the original sample data, $S$ is obtained. Here $\mathrm{S}$ is consisted of $6^{*}\left(10^{* 4}\right)$ data.

\section{(II) Normalization}

Using formulas in Section 3.1 for positive and negative attributes to normalize, package losing rate normalization is show in Fig.2.

\section{(III) Construction of projection index}

One-dimensional pr ojection could be de scribed as formula (9). Two-dimension projection, as formula (10), is more complex than one-dimension projection. However, $t$ wo-projection rem ains more i nformation so it's more accurate.

\section{(IV) GA Searching}

Our platform is M ATLAB 7. 9. M ATLAB G A toolbox is taken in $t$ o solv e the problem. To find the optimal projection mechanism, we adopt three methods: MI, project respectively according to each moment then process condensed projection; MII, one-dimension with time characteristics togeth er; MIII, visualized twodimension projection, shown as following.

\subsection{Experiment results}

MI has two processes: project at each $\mathrm{m}$ oment and then condensed-project for previous values on the time axis. Firstly, use SGA to obtain opti mal proj ection directions of the ten $m$ oments in the first stage shown in Fig. 3. The optimal projection result is showed in Fig. 4.

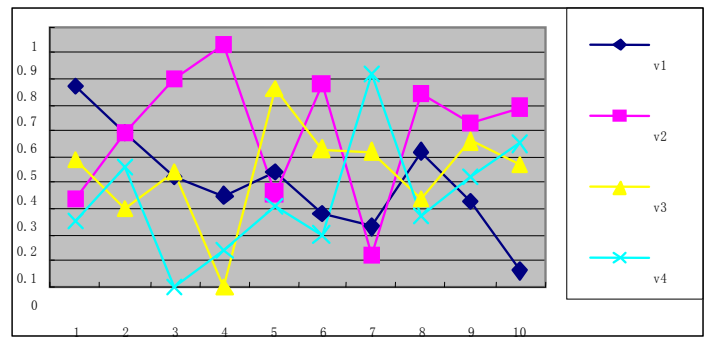

Fig.3. Optimal projection directions of the ten moments 


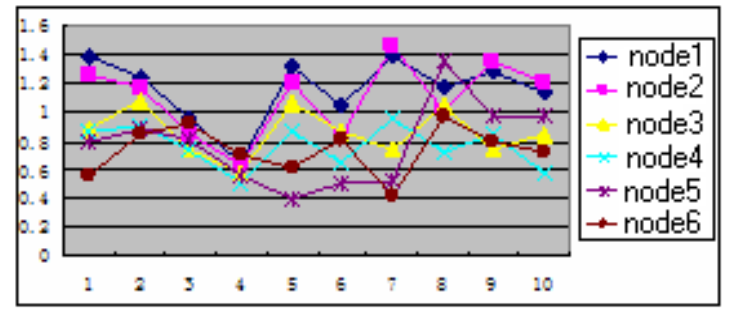

Fig.4. The optimal projection result

Next, project above results on the time axis, the final results is in Fig.5. Nodes 4 and 5 have lower values so they are more risky, PPRA concludes that they are lowefficient or ha ving low trust level. Fig. 6 shows that fitness is relatively convergent in this mechanism.

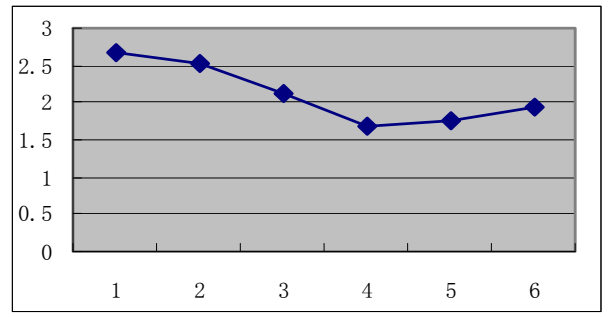

Fig.5. One-dimension projection for MI

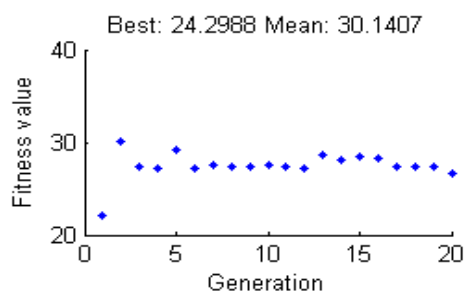

Fig.6. Convergent of one-dimension projection

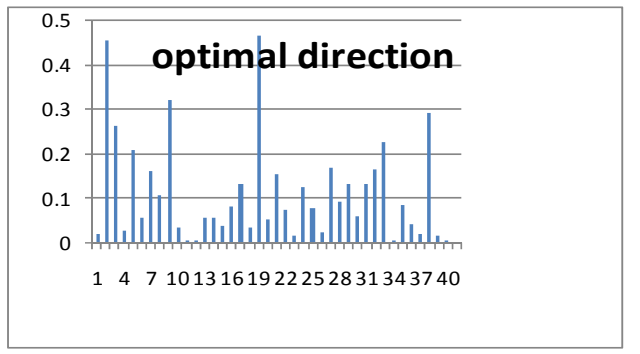

Fig.7. Optimal direction in one-dimension projection

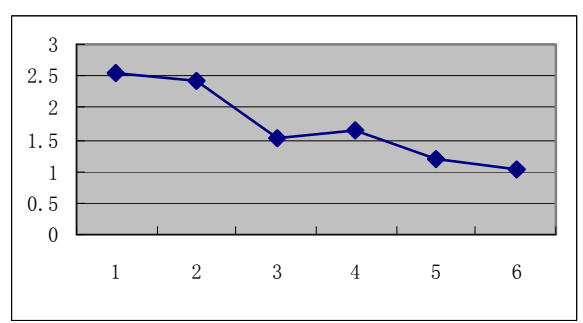

Fig.8. One-dimension projection for MII

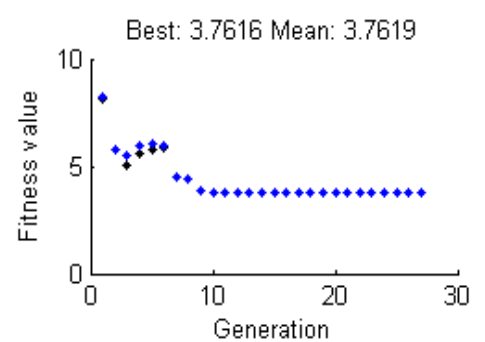

Fig.9. Convergent of one-dimension projection

In MII, with the help of GA, the optimal 40 dimension projection direction is obtained, presented in Fig.7. Given $\boldsymbol{Z}$, the risk level could be calculated using formula (6), shown in Fig.8. Th e first and s econd nodes a re the best. Moreover, the distance between them is quite small, so they are t hought to have sim ilar properties. And node 5 is vulnerable so its projection value is quite small. Meanwhile node 6 is a low-efficiency node; its projection value is sm all too. Fi g.9 shows that the fitness value is relatively converge nt. From the above experiments, we can see the goals of the two mechanisms are consistent. However, MII has better projection result than MI, because MI has two stages, and during the first stage some time characteristics of attack behaviors have been lost already, which expands the gap from the reality.

Even though the result is relatively satisfying, from one-dimension projection, a plenty of information is lost. For example, in o ne-dimension projection both node 5 and node 6 are $b$ ad nod es and it' $s$ hard to d ifferentiate them. $\mathrm{M}$ oreover, one-dimension projection ha $\mathrm{s}$ wea $\mathrm{k}$ visibility. So i ntroducing t wo-dimension proj ection is quite significant.

As for the two-dimension projection, the experiment result is showed in Fig.10. In Fig.10 there are four categories. Node 1 and node 2 belong to the relative good nodes, while the projection value of node 5 is quite low and so as the node 6 . What's more, node 5 is quite different from node 6 . T he re sults accuratel y match our analysis to the ori ginal data. Fig.11 shows th e convergent of two-dimension projection and it's the certification of our model's correctness.

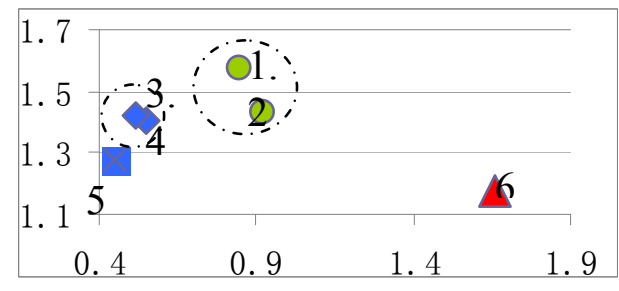

Fig.10. Two-dimension projection value 


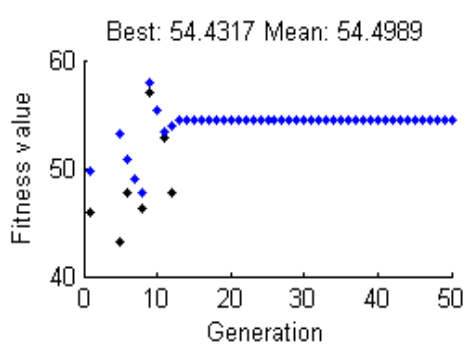

Fig.11. Convergent of two-dimension projection

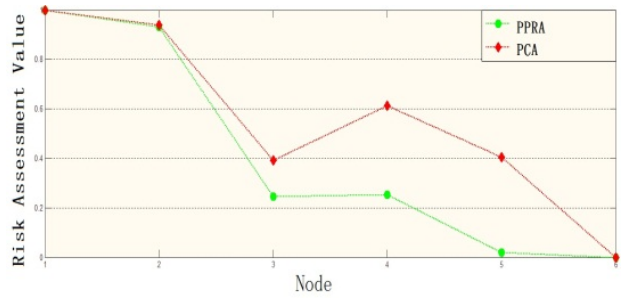

Fig.12. Compare of PP and PCA

\section{Performance Analysis}

\subsection{Projection effectiveness}

We have showed the fitness of $\mathrm{c}$ onvergence. Now we would like to ex plain the reason. In the analysis of PP effectiveness, we mainly depend on the convergence of SGA algorithm. The most difficult problem in GA is to prove the convergence process. Usually, we adopt the method of a nalyzing the ev olution process of optimal function values, so we list the figures of convergence of optimal function values. The convergent process is o bvious in these figures.

\subsection{Data mining experience-irrelevance}

With the nodes in MANET, their attributes are highdimensional a nd variable, from this as pect, dy namic trust ev aluation based on pro jection pur suit can be regarded as a kin d of data mining base do $\mathrm{n}$ hig $\mathrm{h}$ dimensional a nd variable data. Through th e establis hment and use of PP model, we can project any multipledimension dat a which repres ent $m$ ultiple attributes of nodes in MANET to one-dimension space, so as to obtain the only trust attribute and finish the t rust evaluation. $\mathrm{T}$ he data in the eval uation $\mathrm{p}$ rocess $\mathrm{c}$ omes from multiple-attribute and dynamic values ba sed on n odes behaviors.

The drawback of traditional mechanism involves the massive attributes they need and the large data must fit some particular distributions suc $\mathrm{h}$ as beta an $\mathrm{d} \mathrm{B}$ ayes. While for our model, high-dimension dynamic data can be disp osed before projection, so that we do not need massive data for analysis. Even better, a little information can finish the evaluation and we do not need the attributes to accord with any experience probability distribution. Our $\mathrm{m}$ odel is applicable for any highdimension dy namic data and has pretty go od experience-irrelevance. Traditional evaluation need to preprocess the data, while in our model, even without pretreatment, suitable proj ection ind ex an d projection direction can ensure the acc uracy of the result. Therefore, RRPA has litt le requirem ents for data and owns good robustness.

\subsection{Accuracy of Risk Assessment}

The quantization value from PPRA can appropriately reflects trust inform ation of nodes, especially the cluster status from visualized clustering projection, so that we ca $n$ judge whethe $r$ the $n$ odes suffer from the grey ho le attack or DoS attack and so on. Our model does not depend on experience probability distribution and has more accuracy. Traditional evaluation $\mathrm{m}$ echanisms choose a few principal components as evaluation index or p rocess com prehensive jud gment. Pre viously, the risk value has been de fined which actually damage the o riginal a mount of in formation. While o ur m odel bases on the original data so there is no impair or influence for the analysis. The result is certainly more accurate.

To illustrate the advantage, we provide an exam ple to compare our model with Principal Component Analysis (PCA)

In view of the above 6 nodes, 4 attributes in 10 different moments of distribution, use PC A to cluster for evaluation then obtain the following 6 evaluation values: $\mathrm{Y}^{P C A}=\left[Y_{1}^{P C A}, Y_{2}^{P C A}, Y_{3}^{P C A}, Y_{4}^{P C A}, Y_{5}^{P C A}, Y_{6}^{P C A}\right]$

$=[1.0000,0.9404,0.3916,0.6129,0.4041,0]$

Compare this to Fig.12, we find that Node 4 is more risky than Node 5 in PC A, while in actual original data, Node 5 , as a suspicious node, its risk value should near to Node 6'. This result indicates that there exists erroneous judgment of PCA algorithm. PCA is based on mathematic model and its expandability is very limited, especially in dealing with the high-dimension data. Comparably, projection pursuit can search the optimal direction to reduce dimensions. This kind of method considers the inner connection am ong data so the information loss is very little. We can conclude from the figures that the evaluation value from PPRA is much better than that from PCA. PCA $m$ ay even reduce som e effective information and rely only on a few principal components. 
Our m odel ul timately saves the original information. From above results, we can find out that PCA ig nores the dangerous node 5 which conflicts the fact.

\subsection{Time complexity of PPRA}

The a nalysis of time co mplexity should be considered from two aspects: one is without optimal direction; the other is with the optimal direction.

If we do not have optimal direction, GA is necessary. The time complexity of PPRA mainly includes $Q(a)$.

For one-dimension a nd $t$ wo-dimension projection, the total dispersion com plexity of pr ojection data is $O(n m), n$ indicates the amount of nodes and $m$ indicates the dimensions of vector space

$$
O(\mathrm{~s}(\mathrm{a}))=\left\{\begin{array}{c}
O(n * m) \text { one }-\operatorname{dim} \text { ension } \\
O\left(n *\left(O\left(d\left(y_{a}\right)+d\left(y_{b}\right)\right)\right)\right)=O(n * m) t w o-\operatorname{dim} \text { ension }
\end{array} .\right.
$$

The calculation method of local density is:

$$
O(\mathrm{D}(\mathrm{a}))=\mathrm{n} * \mathrm{n} * O\left(\mathrm{r}_{i k}\right)=O\left(n^{2} m\right) \text {. }
$$

Moreover, the com plexity of GA is proportional to the am ount of projection directions of initial subgroup (k), so that the time complexity of GA is:

$O(\mathrm{Q}(\mathrm{a}))=O(\mathrm{~S}(\mathrm{a})+\mathrm{D}(\mathrm{a})) * \mathrm{k}=O\left(n^{2} m k\right)$.

About the spa ce com plexity, because a few subgroups are produced which include projection directions, the com plexity is: $O(w m k), w$ indicates the objective projection dimensions (in this paper, $w=1$ or $w=2$ ). And the space of original data is $O(n m T), T$ is the time point for sampling (here, $T=10$ ).

If we have obtained the projection index and optimal projection direction for a special kind of attack through training with massive sam ple data, the evaluation just needs som e sim ple matrix calculations to obtain the nodes' trust level. Under such circumstances, time complexity is $O(n)$. E.g., for the grey hole attack, we define projection index as $Q(a)=S z * D z$ and the optimal projection direction $a$. We adopt the obtaine d optimal direction to calculate the proj ection values of any node: a(node 1 ), a(node 2 ), ..., a(node $n$ ), by analy zing the trust level, so we can decide whether or not to include some nodes in the routing of self-organize networks.

\subsection{Analysis for realization mechanism}

According to the data $\mathrm{f}$ rom stim ulation u nder the gray hole attack, we obtained the sample of 6 nodes and 4 relevant attributes in 10 different moments. If the projection index is given, we have dif ferent projection methods to get the optimal projection direction for evaluating node risk.

(a) Firstly reduce the dim ensions of the four attributes of each node in every time point. For each tim e point, we establish a $6 * 4 \mathrm{~m}$ atrix, including all attributes of the six nodes. After $\mathrm{p}$ rojection, f our a ttributes be come a single com prehensive value. Apply this m ethod to each node, and then we obtain si $\mathrm{x} \mathrm{c}$ omprehensive values. Synthesizing ten $m$ oments, use the re ducingdimensions process again, attributes in ten moments are projected to one-dimensional space. This can produce an unique trust value for each node.

(b) There a re forty releva nt da ta for each node, reduce dimensions at the sa me tim e, projecting these forty data to one-dimensional space. It deals with a $6 * 40$ matrix directly. Applying the same projection index, we obtain the projection in dex through PP, which re flects the $\mathrm{c}$ haracteristics of all attributes $\mathrm{i} n \mathrm{t}$ he whole tim $\mathrm{e}$ domain.

(c) Project hi gh-dimension data $\mathrm{t}$ o two dimensional space, as form ula (10). MI adopts onedimension projection and projects ten moments respectively. This can lead to the 1 oss of information that expends the ga $p$ with fact. MII adopts direct onedimension projection and combined time characteristics with samples. The result is relatively accurate after optimizing pro jection direction. MII I not on ly co nsiders the time characteristics but a lso reduces the loss of information. So two-dimension projection is more accurate than one-dimension, but the calculation is $\mathrm{m}$ ore complex. Therefore, whe $\mathrm{n}$ c alculating the risk val ues, we usually adopt MII, however, if the data is extraordinarily suspicious, we can co mbine with MIII to process visualized risk evaluation in order to dig out abnormal nodes which may exert attacks.

\subsection{Comparison with other methods}

PPRA model has advantages in node risk assessment for MANETs, as following:

First, compared with reputation-based and probability-based models, PPRA model is "data-driven" without presumed requirement to sam ple data. Even the sam ple data is noisy, PPRA model could be accurate as both the projection in dex an direction a re well $\mathrm{c}$ hosen. While in Ref.3, the sample data must obey the beta distribution, so in the case that the distri bution cannot be satisfied, the assessment result won't be accurate.

Second, P PRA is a visual risk asse ssment m ethod. Risk level is not sim ply cal culated, but clustered and 
visualized. We turn the risk calculation int o risk level clustering and the final results prove our idea is practical and reliable.

Third, PPRA model could be applied to node behavior's analysis. Only a little calculation would be required if optimal projection inde $\mathrm{x}$ an d direction under special condition are found. As we known, the behavior of node in M ANETs is quite complex, but behind the complexity there is simplicity. Although the data is huge, corresponding projection index and direction aiming at ce rtain node's specific behavior can be found by supervised learning; and analysis of its behaviors will be proper after quite easy calcula tion. For example, for the gray hole attack, if the behavior's projection direction is obtained, judgment concerning whether the node is under the gray hole attack or not can be made on the basis of cor responding pr ojection values. Moreover, only simple matrix calculation is needed.

\section{Conclusion}

In this paper, a new model is proposed to deal with the following problems for nodes' risk assessment:

(1) Indicators are high dimensional and multi-polar;

(2) Node's behavior is complex and caprice. Using a comprehensive method to analyze is challenging.

The merits of our proposed schem e could be s ummarized as follows:

(1) There is no restriction for sample data.

(2) PPRA model is simple and does not need complex calculation. How to define more suitable projection index and projection direction for node behavior's analysis remains to be solved in the future.

(3) Our risk a ssessment is $\mathrm{m}$ ore accurate than $\mathrm{t}$ he traditional methods for the reason that the optimal projection direction in PPRA is a comprehensive and effective way to measure nodes' multiple-attributes.

The final result shows the PPRA m odel is effective and $\mathrm{s}$ uitable for risk asses sment in mobile ad hoc network.

\section{Further Work}

Our model is elementary but useful. In our future work specific network behaviors will be analyzed, such as gray hole attack, how to construct corresponding projection index and what's the optimum direction to discriminate nodes under various conditions is of significance.

\section{Acknowledgment}

This paper is supported by the Provincial Natural Science Fo undation of $\mathrm{C}$ hina Hubei (2 008CDB352) an $\mathrm{d}$ the National Natural Science Fo undation of $\mathrm{C}$ hina (60903175 and 60903196).

\section{References}

1. I. Chlam tac, M . Conti, and J.J.N. Liu. Mobile a d hoc networking: im peratives and challenges. Ad Hoc Network, vol .1, pp. 13-64, 2003.

2. Beth T., Borch erding M., Kle in B. Va luation of trust in open netw orks. In P roceeding of European $\mathrm{S}$ ymposium on Research in Security, pp.3-18, 1994.

3. S. Ga neriwal, L.K. Ba lzano, M.B. Sriva stava. Re putation-based framework for high integrity sensor networks. ACM Transactions on Sensor Networks, vol.4 , pp.1-5, 2008.

4. Ye AYong, Ma JianFeng, A tr ust valuation m odel in MANET. Journal of Computer Resear ch and Development (Chinese), 45(5)(2008), pp.765-770.

5. Z. Qin, Z. Jia, X. Che n. Fuzzy d ynamic progr amming based trusted routing decision in mobile Ad $\mathrm{H}$ oc Networks. Em bedded Com puting, SEC '08 . F ifth I EEE International Symposium, pp.180-185.

6. Tang W., Chen Z. Res earch of subjective trust management model based on the fuzzy set theory. Chinese Journal of Software, pp.1401-1408, 2003.

7. J.H. Friedman and J.W.Tukey. A projection pursuit algorithm for exploratory data analysis. IEEE Transcaction on Computers, September, 1973.

8. Liu M, Gong H. A distributed energy-efficient data gathering and aggr egation proto col for wireless sen sor networks. Chinese Journal of Software, vo 1.16, 2106-2116, 2005.

9. Heinzelman W.R. An application-specific protocol architecture for $\mathrm{w}$ ireless microsensor networks. IEEE Transc. on Wireless Communications, vol.1, pp.660-670, 2002.

10. J. Hwang, S. Lay, M. Maechler, R.D. Martin. Regression modeling in $\mathrm{b}$ ack-propagation and projectio $\mathrm{n}$ pursuit learning. IEEE Transaction on Neural Networks, 1994.

11. F. Qiang, Zhao X. Project pursuit theory and application. Chinese Science Press, pp.6:12-28, 2006.

12. Devinder Kaur, Praneeth Ne lapati. Perform ance enhancement of $\mathrm{d}$ ata classification using selectively cloned genetic algorithm for neural network. International Journal of Computational Intelligence Systems,3(6)(2010), pp. 723-732. 